\section{Visual masking and unconscious processing: Differences between backward and simultaneous masking?}

\author{
ANTHONY G. GREENWALD and MARK R. KLINGER \\ University of Washington, Seattle, Washington
}

Visual masking procedures are considered to have great potential for studying information processing that occurs outside of consciousness. Unfortunately, effects that indicate processing of masked word stimuli have been both difficult to obtain and, once obtained, difficult to replicate. The present seven experiments failed to obtain an effect of lexicality (word vs. nonword targets) on detection that was recently reported by Doyle and Leach (1988). Whereas Doyle and Leach had used backward binocular masking, most of the present experiments used simultaneous dichoptic masking. Doyle (1990) recently suggested that the effect of lexicality on detection (coupled with an effect of knowledge of results, which was also not obtained in the present research) could explain why Greenwald, Klinger, and Liu (1989) found no evidence for detectability of masked words that were nevertheless analyzed semantically. The differences of the present findings from those of Doyle and Leach (1988) not only confirm the uncertainty of generalizing across masking procedures, but also indicate that Greenwald et al.'s "detectionless processing" interpretation remains viable.

Holender's (1986) review of "semantic activation without conscious identification" (abbreviated SA/CI) focused attention on claims that visually pattern-masked words can produce SA/CI. Holender interpreted the existing evidence for such claims as unconvincing, but he also noted that the procedure of visual pattern masking had substantial potential, on theoretical grounds, to demonstrate SA/CI. In the period since Holender's review, two trends can be identified in studies that have used visual masking procedures to study unconscious processing.

SA/Cl at subjective thresholds. Cheesman and Merikle (1985) suggested that SA/CI (1) can be obtained replicably under "subjective threshold" masking conditions, which afford some detection and identification of words even though subjects claim not to see the words, but (2) cannot be obtained under "objective threshold" conditions that effectively eliminate detectability. This view has been favorably received, no doubt partly because it provides a rapprochement of empirical findings of SA/CI with established information processing theories. Consistent with Cheesman and Merikle's analysis are studies that have produced SA/CI findings under conditions that are likely to permit low levels of detection (e.g., Bargh \& Pietromonaco, 1982; Kitayama, 1990; Reingold \& Meri$\mathrm{kle}$, in press). Also consistent with Cheesman and Meri-

\footnotetext{
This research was supported by National Institute of Mental Health Grant MH-41328, and by a National Institute of Health Biomedical Research Support PHS Grant RR-07096. Correspondence may be addressed to Anthony G. Greenwald, Department of Psychology, NI-25, University of Washington, Seattle, WA 98195.
}

kle's view are studies that have obtained evidence for unconscious processing of nonword (e.g., shape or face) stimuli under conditions that likely afford some detection, but not enough for stimulus identification (e.g., Bonanno \& Stillings, 1986; Bornstein, Leone, \& Galley, 1987; Lewicki, 1986; Mandler, Nakamura, \& Van Zandt, 1987; Seamon, Brody, \& Kauff, 1983).

$S A / C I$ at objective thresholds. A few studies have claimed to produce $\mathrm{SA} / \mathrm{CI}$ findings under masking conditions that do not permit detection of word stimuli (Dagenbach, Carr, \& Wilhelmsen, 1989; Greenwald \& Klinger, 1989; Greenwald, Klinger, \& Liu, 1989). A problem for this second group of studies is the difficulty of establishing objective threshold levels due to the statistical difficulty of accepting a null hypothesis (i.e., that detectability is at chance). ${ }^{1}$

The preceding article by Doyle (1990) enters this empirical debate on Cheesman and Merikle's side, suggesting that Greenwald et al.'s (1989) claimed objective threshold presentations were actually at subjective thresholds. Doyle made use of the results of Doyle and Leach (1988), who showed that detection of masked stimuli was facilitated both by providing the subject frequently with knowledge of results (KR: feedback on detection accuracy) and by using words (rather than nonsense strings) as target stimuli for detection tests.

To test for detectability of masked stimuli, Doyle applied his analysis to the position-discrimination task used by Greenwald et al. In that task, subjects judged on each trial whether words were presented to the left or right of a centered fixation point. Doyle noted that, if Greenwald et al.'s subjects were operating at subjective rather than objective thresholds (i.e., if their detection was above chance rather than at chance), Greenwald et al. could have observed performance impaired to near chance because (1) frequent KR was not used and (2) the semantic content of target stimuli frequently conflicted with the position dimension that was to be judged. (Target stimuli were the words LEFT and RIGHT, the meanings of which conflicted with their locations on half the trials). ${ }^{2}$

This article presents experiments showing that, under the simultaneous dichoptic masking conditions used by Greenwald et al. (1989), the beneficial effects on detection of KR and lexicality found by Doyle and Leach (1988)-who used backward binocular masking-are not obtained.

\section{GENERAL METHOD}

With the exception of Experiment 7 (which used different masking procedures from Experiments 1-6), the experiments used largely similar procedures, which are described in this section. Table 1 provides some details of procedures that varied across experiments.

\section{Subjects}

The participants in Experiments 1-5 were male and female volunteers who received credit toward a grade in their introductory psy- 


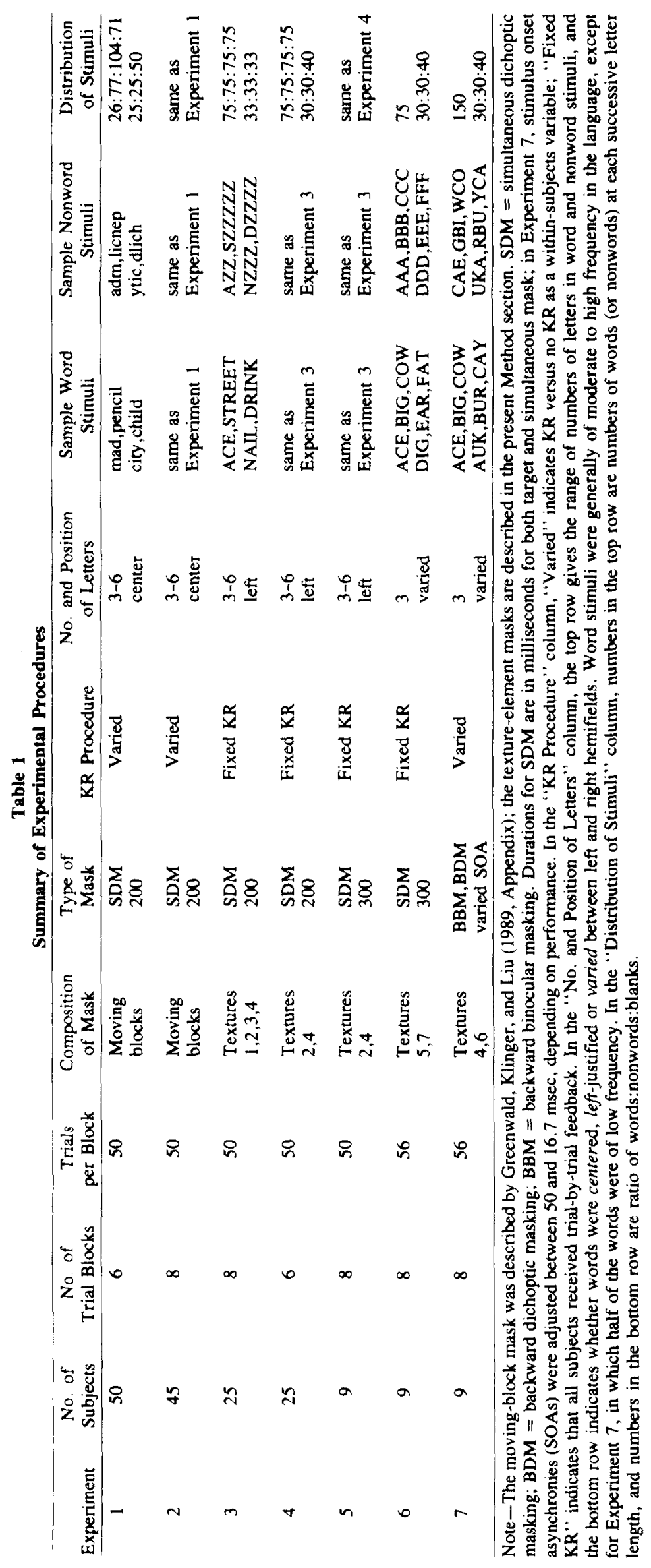


chology course. Experiments 6 and 7 each used the same group of 9 subjects, 6 of whom were paid volunteers recruited via an advertisement in the campus newspaper at the University of Washington; the others were the two authors and a graduate student volunteer. The subjects were required to be fluent in English and to have normal or corrected-to-normal vision. The analyses reported here included data for all subjects. Additional analyses, excluding those subjects for whom masks approached complete effectiveness or complete ineffectiveness, presented the same picture as the ones reported.

\section{Apparatus}

The subjects viewed a computer display from $65 \mathrm{~cm}$, the length of a rectangular (cross-section) viewing tube that had a central vertical divider, enabling dichoptic presentations (separate stimuli to each eye). The display was driven by an enhanced graphics adapter (EGA) video interface on a PC/AT-type computer. Rotary prisms, at the subject's end of the viewing apparatus, superimposed the images to each eye. (This apparatus was previously described by Cheesman \& Merikle, 1986.)

\section{Detection Task}

The subjects were informed that a letter string would be presented on a random half of the trials. They were to press one keyboard key to indicate "yes" (letter string present) and another to indicate "no." The subjects received trial-by-trial feedback during about 50 trials of practice. (This use of feedback was intended to increase the rate of "yes" responses from those subjects for whom the mask was highly effective.) If a subject exceeded $70 \%$ correct for these practice trials or for any subsequent block of detection trials, contrast in the target field was reduced. These adjustments were made so that the subjects would perform well below $100 \%$ correct, but nevertheless enough above chance to permit observation of treatment effects.

\section{Masks}

The mask for Experiments 1 and 2 used a pattern that was constructed from graphics elements available in the standard IBM/PC display character set (see Figure 1, upper left panel). This mask was presented with its middle row of characters shifted one character position to the right every $67 \mathrm{msec}$, creating the appearance of move- ment. For Experiments 3-7, masks were composed of fabricated display characters, constructed by placing pixels at selected positions in the 8 (horizontal) $\times 14$ (vertical) dot matrix that comprises a character representation for the EGA-interface display. The fabricated characters were constructed so that, with appropriate sideby-side and top-to-bottom juxtapositions, regularly spaced gratings oriented vertically, horizontally, or in either diagonal direction could be constructed, with thickness of the parallel lines and spaces ranging from 1 to 7 pixels. However, rather than using such grating-like masks, the masks were constructed by randomly selecting on each trial, for each position in a 3 row $\times 15$ column rectangular array, elements corresponding to a selected thickness. ${ }^{3}$ Sample masks at element thicknesses of 3,5 , and 7 are shown in Figure 1.

\section{Target Stimuli}

Each experiment included both word and nonword targets. Words were typically of moderate to high word frequency (which, following Doyle \& Leach, 1988, was expected to maximize the likelihood of word-nonword differences). Nonwords were composed in varying fashion (see Table 1) but were always matched with words for case, length, initial letter, and position in the display. ${ }^{4}$ The stimulus (word, nonword, or blank) used on each trial was selected randomly (without replacement for words and nonwords in most of the experiments). Despite variations in the proportion of blank stimuli across experiments (see Table 1, far right column), the subjects were instructed in all experiments that blanks and letter strings were approximately equally frequent in occurrence. Nonblank trials were equally likely to be words or nonwords.

\section{Knowledge of Results}

Trial-by-trial feedback (KR) was used for all blocks of trials in Experiments 3-6. However, in Experiments 1, 2, and 7, KR was a within-subjects variable. Order of blocks of trials with and without KR was counterbalanced across subjects. In Experiment 2, all blocks in the same $\mathrm{KR}$ condition were consecutive, permitting a betweensubjects test of the effect of KR that used only the first condition to which each subject was exposed. On each trial in a block with KR, the word "error" was presented after each miss or false alarm. The subjects also always received block-end reports of their percent correct at detection in the preceding block. (In this sense, there

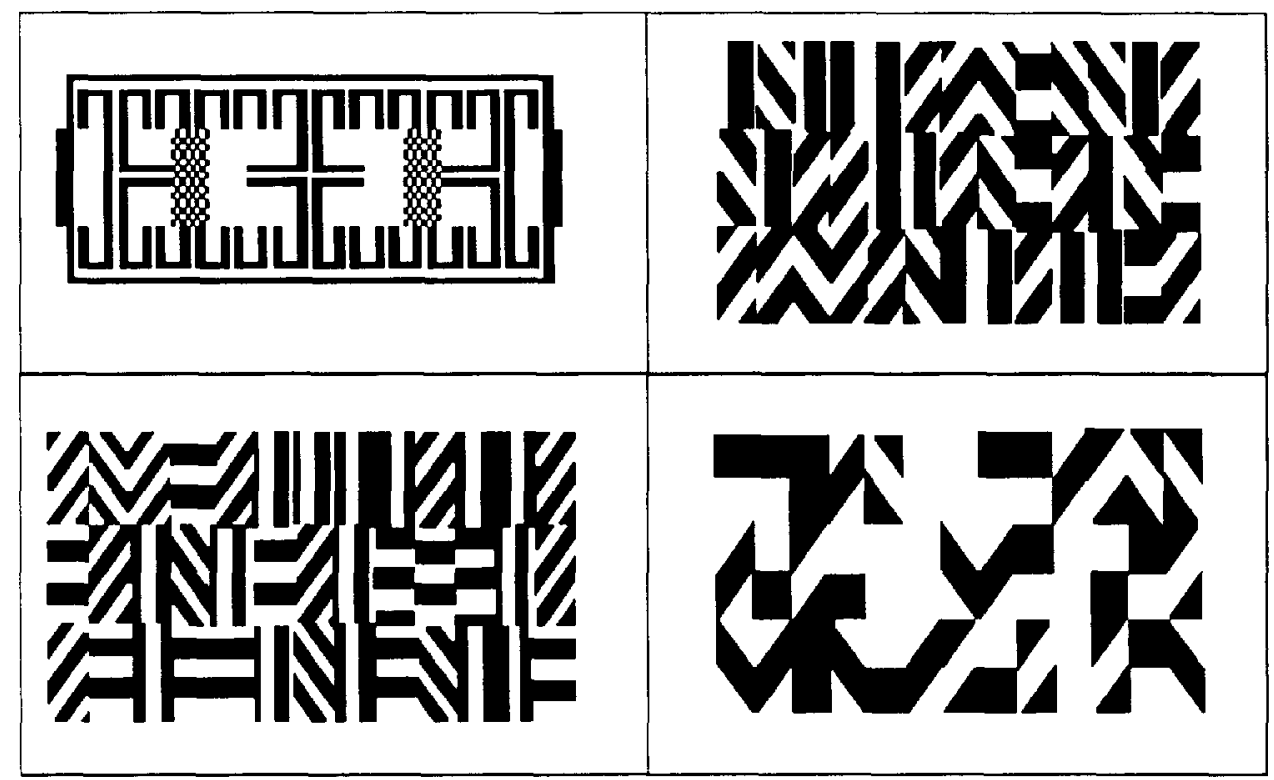

Figure 1. Sample visual masks. (See text for further description.) 
was KR every 50 or 56 trials even during no-KR treatments; however, this infrequent feedback was assumed to constitute at most a minor contamination of the KR procedure.)

\section{RESULTS}

\section{Measures of Detection}

Hit-rate $(H)$ and false-alarm-rate $(F A)$ values occasionally reached their extremes of 0.0 or 1.0 , rendering the signal detection theory measure of sensitivity, $d^{\prime}$, noncomputable. Tests of effects of independent variables were therefore made on three nonparametric measures of sensitivity: $(H-F A) ;(H-F A) /(1.0-F A)$, which modifies the first index by incorporating a correction for guessing; and gamma $=(H-F A) /[H+F A-2(H)(F A)]$ (see Nelson, 1986). The variations in patterns of results across the three sets of analyses were too minor to warrant any differences in conclusions. We report the results for the simplest index $(H-F A)$, which also had the lowest variability across subjects. ${ }^{5}$

\section{Effect of KR on Detectability}

Experiments 1, 2, and 7 varied $\mathrm{KR}$ within subjects. Figure 2 presents the results. For the $(H-F A)$ detectability measure (upper panel of Figure 2), all three of these experiments indicated (contrary to Doyle \& Leach, 1988) slightly worse performance with KR than with no
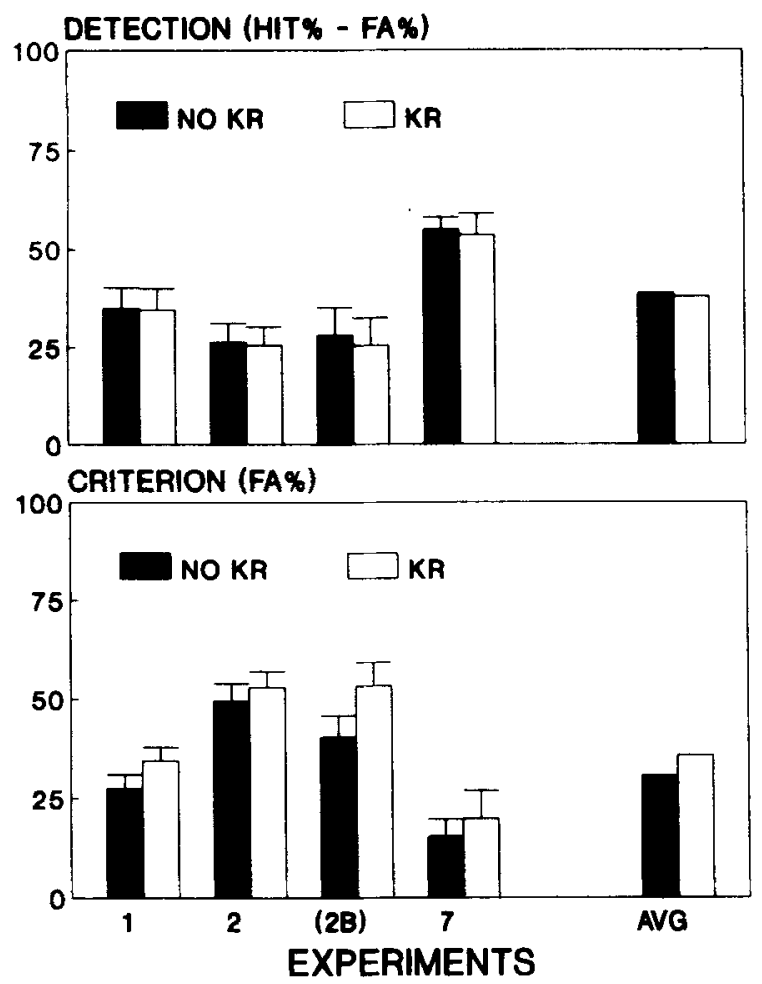

Figure 2. Effect of KR on detection (hit rate minus false-alarm rate) and criterion (false alarms). The average data combine the within-subjects data of the three experiments. The between-subjects data of Experiment 2 (first condition done by each subject) are also shown (as Experiment 2B).

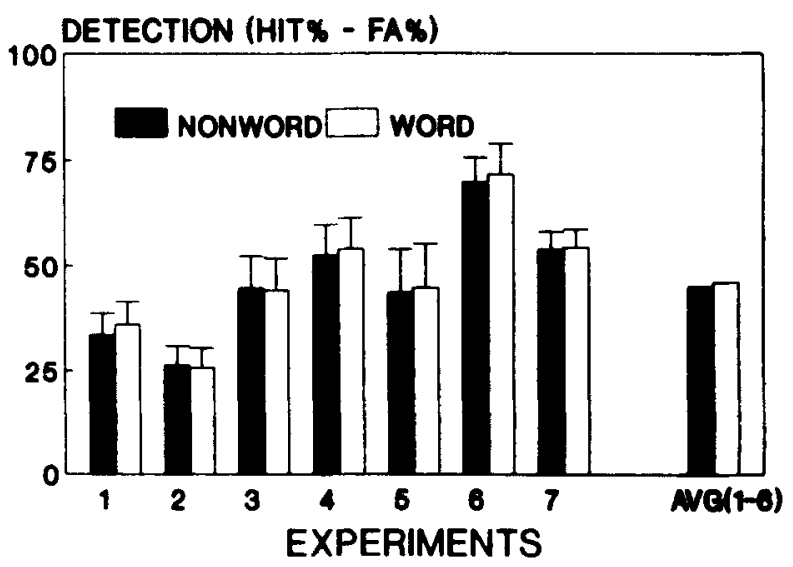

Figure 3. Detection of words versus nonwords (hit rate minus falsealarm rate). The average data combine the six experiments that used simultaneous dichoptic masking (Experiments 1-6).

KR (all $F$ ratios $<1$ ). The lower panel of Figure 2 shows, however, that false-alarm rates were higher with $\mathrm{KR}$ than with no KR in all three experiments; this effect was statistically significant in Experiment $1[F(1,49)=$ $7.62, p<.01]$ but not in Experiments 2 or 7 . A combined test of significance across the three experiments (Stouffer method, see Mosteller \& Bush, 1954) produced a $z$ of $2.73(p<.01)$, indicating that the greater falsealarm rate for the KR condition was statistically significant.

Experiment 2 additionally permitted a between-subjects test of the effect of KR, using data from each subject's first four blocks of detection trials; 23 subjects received $\mathrm{KR}$ first and 22 received no KR first. Again, detectability $(H-F A)$ was slightly better with no $\mathrm{KR}(M=28.0 \%)$ than with $\mathrm{KR}(M=25.4 \%)$, and again the false-alarm rate was higher with $\mathrm{KR}(M=53.4 \%)$ than without $\mathrm{KR}$ $(M=40.4 \%)$. However, neither of these results was statistically significant $[F(1,43)<1$, and $F(1,43)=2.58$, respectively].

\section{Detectability of Words versus Nonwords}

Figure 3 presents the detection data for words and nonwords in Experiments 1-7. Words were more detectable than were nonwords in five of the seven experiments, but the differences were generally quite small and were not significant in any experiment (all $F$ s between 0.08 and 2.16). The result of a combined test of significance (Stouffer method) of the six experiments that used simultaneous dichoptic masking (Experiments 1-6) yielded a $z$ of $1.56(p>.10)$, indicating a nonsignificant combined effect (which was, however, in the direction of greater detectability of words than nonwords).

Experiment 7 was distinctive in using backward masking rather than simultaneous masking and also in using word frequency as an independent variable. Both of these changes from the previous experiments were instituted in order to have procedures more similar to those used by Doyle and Leach (1988). In the procedure of Experi- 
ment 7, backward dichoptically masked trials and backward binocularly masked trials were randomly mixed within blocks. Because there were no differences in effects when the two types of trials were analyzed separately, the results will be described only for an overall analysis. There was no difference in detectability for words (overall) versus nonwords $(F<1)$. However, there was an effect of word frequency on detectability. On the $(H-F A)$ measure, high-frequency words $(M=57.4)$ were detected at a higher rate than were lowfrequency words $[M=51.4 ; F(1,8)=6.38, p<.05]$ and were also detected more than were nonwords $(M=53.9)$, although the latter difference was not statistically significant $[F(1,8)=1.99]$.

\section{DISCUSSION}

The results of the present experiments did not confirm the conclusions reached by Doyle and Leach (1988) regarding the effect of either KR or target lexicality on detection of visually masked letter strings. In particular, the present results indicated that (1) detection performance was not improved by KR relative to no KR and (2) masked words were no more detectable than were masked nonwords. Two additional findings are important in considering the relation of our results to the conclusions reached by Doyle and Leach.

First, although KR had no effect on our measures of detectability, it did have a reliable effect on false alarms, such that the criterion for a "yes" response was lower when KR was provided-the subjects were more willing to guess that a target was present when they received frequent KR. Under conditions of low detectability, this effect on criterion could masquerade as an effect on detectability; subjects who rarely or never guess that a target is present will be at or near the floor on most computable measures of detectability. For these subjects, relaxation of criterion (guessing "yes" more often) will improve detection scores, assuming that the added "yes" responses are more likely to occur on signal-present trials than on signal-absent trials. Doyle and Leach (1988) used a twoalternative discrimination task (target before first mask vs. second mask) rather than presence-absence detection. Consequently, this interpretation could explain their effect of KR on detection only if their no-KR subjects who were detecting poorly tended to guess consistently that the target was in just a single temporal position. ${ }^{6}$

Second, although we repeatedly found no effect of lexicality on detection, in Experiment 7 high-frequency words were more detectable than were low-frequency words. This result raises a question: If high-frequency words are more detectable than are low-frequency words, then why, in Experiments 1-6, were they not also more detectable than nonword letter strings (which are, in effect, at the zero level of word frequency)? This discrepancy, as well as the contrast between our null findings for lexicality and Doyle and Leach's positive findings, might be explained by our shift from simulta- neous dichoptic masking in Experiments 1-6 to backward masking, which was used in our Experiment 7 and also in all of Doyle and Leach's experiments. Possibly, effects of lexicality on detection occur with binocular (and perhaps other types of) backward masking, but not with simultaneous dichoptic masking.

\section{Implications for Interpretation \\ of Greenwald et al. (1989)}

Our findings indicate the need for caution when generalizing Doyle and Leach's conclusions beyond the procedures that they used. In particular, Doyle and Leach's conclusion that words are more detectable than are nonwords does not generalize to the procedures that were used in the present Experiments 1-6. Plausibly, the most significant procedural difference between Doyle and Leach's research and ours is the difference in type of visual masking used-Doyle and Leach used backward binocular masking and we used simultaneous dichoptic masking.

Consequently, Doyle's (1990) suggested reinterpretation of Greenwald et al. (1989)-which assumes a generalization of Doyle and Leach's findings to simultaneous dichoptic masking-loses its empirical basis. We agree, nevertheless, with the spirit of Doyle's comment, which was to seek an interpretation of theoretically puzzling $\mathrm{SA} / \mathrm{CI}$ findings-puzzling because they were obtained under conditions that appeared not to permit detection of stimuli that, nevertheless, received some semantic processing.

Available theories of visual masking (see Breitmeyer, 1986, for a review) do not specify differences between simultaneous dichoptic masking and backward binocular masking that could explain the discrepancy between our present findings and those of Doyle and Leach. Furthermore, because no research has yet compared these two types of masking in a single experiment, it is far from certain that differences in masking procedure are the cause of differences in findings. Nevertheless, it now appears highly desirable for some future studies of $\mathrm{SA} / \mathrm{CI}$ to include type of visual masking as an explicit independent variable.

\section{REFERENCES}

Bargh, J. A., \& Pietromonaco, P. (1982). Automatic information processing and social perception. Joumal of Personality \& Social Psychology, 43, 437-449.

Bonanno, G. A., \& Stillings, N. A. (1986). Preference, familiarity and recognition after repeated exposures to random geometric shapes. American Journal of Psychology, 99, 403-415.

Bornstein, R. F., Leone, D. R., \& Galley, D. J. (1987). The generalizability of subliminal mere exposure effects. Journal of Personality \& Social Psychology, 53, 1070-1079.

BrEITMEYeR, B. G. (1986). Visual masking: An integrative approach. Oxford: Oxford University Press.

Chemsman, J., \& Merukle, P. M. (1985). Word recognition and consciousness. In D. Besner, T. G. Waller, \& G. E. MacKinnon (Eds.), Reading research: Advances in theory and practice (Vol. 5, pp. 311352). New York: Academic Press.

Chemsman, J., Merikle, P. M. (1986). Distinguishing conscious from unconscious perceptual processes. Canadian Journal of Psychology, 40, 343-367. 
Dagenbach, D., Carr, T. H., Wilmelmsen, A. (1989). Taskinduced strategies and near-threshold priming: Conscious influences on unconscious perception. Journal of Memory \& Language, 28. $412-443$.

Doyle, J. R. (1990). Detectionless processing with semantic activation? A footnote to Greenwald, Klinger, and Liu (1989). Memory \& Cognition, 18, 428-429.

DoYLE, J. R., \& LEACH, C. (1988). Word superiority in signal detection: Barely a glimpse yet reading nonetheless. Cognitive Psychology, 20, 283-318

Greenwald, A. G., Klinger, M. R. (1989, November). Further tests for unconscious processing of dichoptically masked words. Paper presented at the annual meeting of the Psychonomic Society, Atlanta, GA.

Greenwald, A. G., Klinger, M. R., \& LiU, T. J. (1989). Unconscious processing of dichoptically masked words. Memory \& Cognition, 17, 35-47

Holender, D. (1986). Semantic activation without conscious identification in dichotic listening, parafoveal vision, and visual masking Behavioral \& Brain Sciences, 9, 1-66.

Kitayama, S. (1990). Interaction between affect and cognition in word recognition. Journal of Personality \& Social Psychology, 58, 209217.

LEWICKI, P. (1986). Nonconscious social information processing. New York: Academic Press.

Mandler, G., Nakamura, Y., \& Van Zandt, B. J. (1987). Nonspecific effects of exposure on stimuli that cannot be recognized. Journal of Experimental Psychology: Learning. Memory, \& Cognition, 13, 646-648

Mosteller, F., \& Bush, R. R. (1954). Selected quantitative techniques In G. Lindzey \& E. Aronson (Eds.), Handbook of social psychology (Vol. 1, pp. 289-334). Reading, MA: Addison-Wesley.

NELSON, T. O. (1986). ROC curves and measures of discrimination accuracy: A reply to Swets. Psychological Bulletin, 100, 128-132

REINGold, E. J., \& MERIKLE, P. M. (in press). Recognition and lexical decision without detection: Unconscious perception? Journal of Experimental Psychology: Human Perception \& Performance.

Seamon, J. G., Brody, N. \& Kauff, D. M. (1983). Affective dis- crimination of stimuli that are not recognized: Effects of shadowing. masking and cerebral laterality. Journal of Experimental Psychology: Learning. Memory, \& Cognition. 9, 544-555.

\section{NOTES}

1. We interpret Dagenbach et al. "s (1989) "detection threshold" as corresponding more to Cheesman and Merikle's concept of objective threshold than to the latter's concept of subjective threshold. Dagenbach et al., it should be noted, acknowledged that such an identification could not be made confidently, and they referred to their presentation conditions cautiously as "near-threshold."

2. By implication, Doyle's analysis also calls into question the characterization of threshold conditions in other studies that did not use KR during detection tests (e.g., Dagenbach et al., 1989; Greenwald \& Klinger, 1989).

3. Thickness of mask elements, which varied within and between experiments, had effects on target detection, such that masks made of elements 5 or 6 pixels thick were most effective (see Greenwald \& Klinger, 1989). Effects of target lexicality and KR were thus observed over a range of masking conditions.

4. Position in the display was found to have effects on detectability (reported by Greenwald \& Klinger, 1989). The most striking such effect was that right-eye-dominant subjects had reduced detection of targets presented to the left hemifield of the left eye (i.e., with a simultaneous mask to the right eye).

5 . The lower variance was associated with greater stability among subjects who were operating at low levels of detection with relatively high false-alarm rates. For these subjects, the two indexes in ratio form had the potential to assume large negative values.

6. Another difference between Doyle and Leach's KR procedure and ours was that we gave KR after every trial, whereas they gave it only after every 10th trial. However, it is difficult to imagine that this difference could have been responsible for their observing an effect of KR while we did not.

(Manuscript received January 29, 1990; revision accepted for publication April 23, 1990.) 